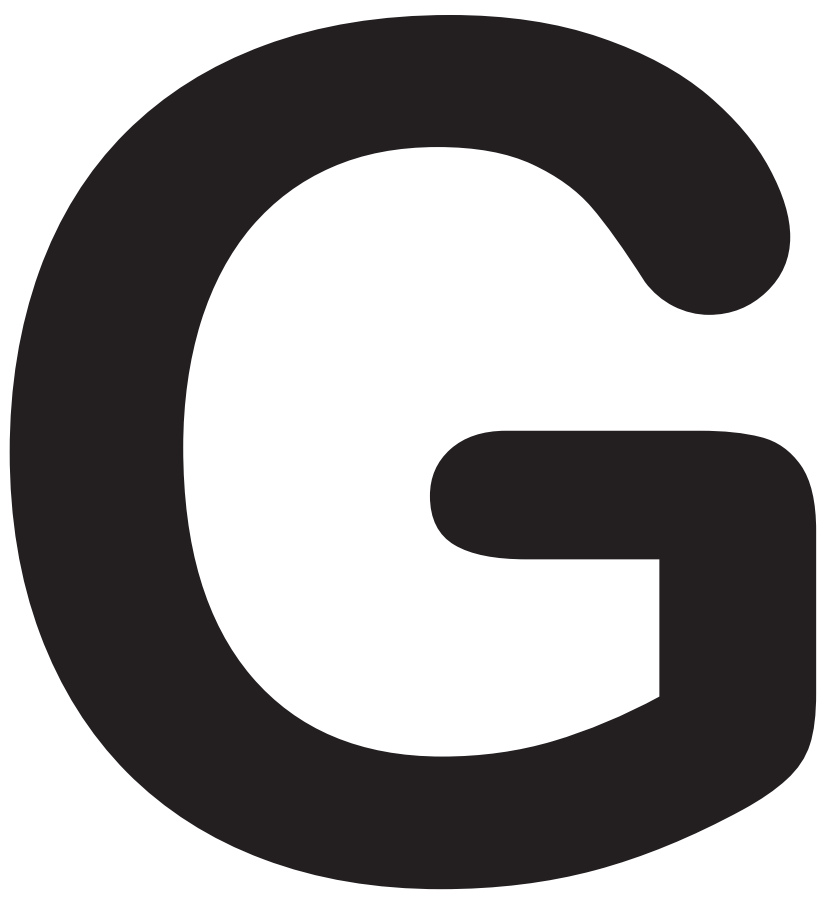

\title{
List of Moderators (G)
}




\section{A}

Abolmaali N.: SS 208

Adam E.J.: RC 714, Standards and Audit

Agadakos E.: SS 214

Allen S.: SS 1702

Almeida P.: SS 609a

Álvarez-Benito M.: SS 1802b

Andersen P.E.: SS 609b

Aparisi Gomez M.P.: SS 1810

Arkhipova I.: SS 203

Aschauer M.A.: SS 115

Avdagic E.: SS 511

Ayuso C.: EM 1

\section{B}

Bakke S.J.: SS 111

Baramia M.: SS 1307

Barentsz J.O.: CC 1421

Bargellini I.: SS 601

Barkhausen J.: SS 515, SY 4

Barsi P.: SS 511

Basile A.: SS 609a

Bauer R.W.: SS 1803

Beale T.: RC 1908

Beer A.J.: SS 1807

Belcari N.: SS 606

Bilbao J.I.: EM 1, EM 2, EM 3, EM 4

Bilecen D.: RC 815

Blackburn Andersen P.: SS 914

Bohata S.: SS 503

Bosmans H.: SS 1813

Brader P.: EIBIR/EORTC Joint Workshop

Brambs H.-J.: RC 401

Brancatelli G.: SS 201b

Brink M.: SS 1317

Brkljacic B.: RC 307

Brountzos E.: CC 1218

Brouwer P.A.: SY 19

Bruegel M.: SS 201b

Bulja D.: TF 1

Buruian M.: SS 1811b

\section{C}

Cáceres J.: RC 304

Cademartiri F.: SS 903

Camps Herrero J.: SY 3

Canet Soulas E.: SS 1803

Cano D.: SS 1701a

Cantisani V.: SS 201a

Carbonaro L.A.: SS 902

Caseiro-Alves F.: SY 6

Castañer E.: SS 104

Catalano C.: MC 722

Chamming's F.: SS 1802a

Chan O.: CC 1418

Choi B.I.: SS 1801b

Cianfoni A.: SS 1811b

Ciraj-Bjelac O.: RC 1113, SS 113

Claudon M.: CC 1521

Claussen C.D.: RC 1514

Clavien P.-A.: EM 2

Clément O.: RC 306

Coenegrachts K.: SS 516

Cotten A.: SS 1810

Cowan N.C.: CC 1121

Crocetti L.: SS 1309

Cunningham P.M.: SS 910

Curvo-Semedo L.: SS 1316

\section{D}

Damilakis J.: ESR Radiation Protection Session

Danielsson M.: SY 16

Das M.: SS 1703

De Foer B.: $E^{3} 420$

De Graaf N.: SS 1712

de Koning H.J.: MC 423

de Roos A.: MC 322

Delorme S.: SS 116

Denecke T.: SS 1716

Denys A.: SS 109

Derchi L.E.: Joint Session of the ESR and EFSUMB

Deutschmann H.A.: RC 715

Di Cesare E.: RC 303

Diederich S.: SS 504

Djilas-Ivanovic D.: SS 502

Donato P.: SS 603b

Dondelinger R.F.: SS 1709

Donoghue V.: RC 1412

Dormagen J.B.: SS 101a

E

Elias R.: SS 1308

Ertl-Wagner B.: SS 111

Esen G.: RC 302

\section{$\mathbf{F}$}

Faggioni L.: SS 205

Faschingbauer R.: SS 101b

Fatehi M.: SS 205

Feragalli B.: SS 117

Ferretti G.R.: SS 204

Feuchtner G.: SS 1303

Filippiadis D.: SS 115

Filippone A.: SS 1801a

Foley S.J.: SS 1714

Fournier L.S.: SS 607

Frija G.: RC 1214

Frühwald-Pallamar J.: SS 608

Fuchsjäger M.H.: CC 1619

Furmanek M.I.: SS 1716

Fütterer J.J.: SS 209

$\overline{\text { G }}$

Garaci F.G.: SS 515

Gennaro G.: SS 602

Gevenois P.A.: RC 1904

Gibaud B.: SS 105

Gilbert F.J.: MC 723

Girard N.: $E^{3}$ 920b

Gizewski E.R.: SS 509

Gleeson F.: RC 1104

Gleeson T.: SS 1806

Golay X.: SS 1311

Góraj B.: SS 611

Gouliamos A.D.: $E^{3} 720 b$

Grainger A.J.: SS 1310

Grazioli L.: SS 1306

Grenier P.A.: SS 904

Gruszczynska K.: SS 1703

Grzycka-Kowalczyk L.: RC 1108

. 


\section{H}

Hagen G.: SS 507

Hakumäki J.: SS 1706

Haller K.: RC 1114

Haller S.: SS 1811a

Hamm B.: SY 18

Hany T.F.: SS 916

Hartley T.: $S Y 1$

Hartmann I.: SS 1304

Healy J.: SS 510

Helbich T.H.: MC 423, SY 5

Hendrikse J.: RC 315

Herold C.J.: SS 216

Herzog C.: SS 1303

Hirsch W.: SS 1312

Hjemly H.H.: SS 1814

Hoeffel C.: SS 1316

Hoeks C.M.A.: SS 507

Houssami N.: MC 623, SS 1302

Hricak H.: MC 528

Huete A.: SS 117

Humphries P.D.: SS 1312

Husty J.: SS 1801a

Jaschke W.R.: SS 215

Johnson K.J.: SS 912

Jung E.M.: SS 1702

\section{K}

Karabulut N.: SS 504

Karpenko A.: SS 610b

Karssemeijer N.: SS 1713

Kellenberger C.J.: RC 812

Kettenbach J.: SS 209

Keyzer C.: SS 216

Kirova-Nedialkova G.I.: SS 903

Klauser A.: SS 210

Koen V.H.: TF 1

Koh D.-M.: CC 819

Kornienko V.N.: SY 20

Koutalonis M.: SS 1713

Kroencke T.J.: SS 101b

Krombach G.A.: SS 103

Kubik-Huch R.A.: SS 202

Kudrnova S.: SS 1809

Kuribayashi S.: SS 1815

Labuscagne J.: SS 910

Lalam R.: SS 610a

Lambregts D.M.J.: SS 916

Lauenstein T.C.: SS 1306

Lehéricy $S$.: $E^{3} 820 b$

Leidecker C.: SS 213

Lencioni R.: CC 1219

Leschka S.: SS 101a

Linsenmaier T.: CC 1618

Liu Y.: EIBIR/EORTC Joint Workshop

Llopis E.: RC 1210

Lonn L.: CC 818

Lopci E.: SS 1706

Lucic M.A.: SS 1812

\begin{tabular}{l} 
M \\
\hline Magnusson A.: RC 707 \\
Maksimović R.: SS $603 b$ \\
Malamateniou C.: RC 1514 \\
Mang T.: SS 905 \\
Marco de Lucas E.: SS 211 \\
Marincek B.: SS 901 \\
Martí-Bonmatí L.: RC 1114 \\
Martinez-Miravete P.: SS 602 \\
Maurer M.H.: SS 1710 \\
McNulty J.: SS 1814 \\
Meaney J.F.M.: CC 1518 \\
Meindl T.: SS 611 \\
Menu Y.: MC 628 \\
Merhemic Z.: RC 1111 \\
Merkle E.M.: SS 1806 \\
Mershina E.A.: SS $603 a$ \\
Mildenberger P.: SS 203 \\
Mileto A.: SS 510 \\
Molyneux A.: E⿱3 620 \\
Morcos S.K.: CC 1621 \\
Morgan R.: SS 1809 \\
Morozov S.: SS 107 \\
Mostbeck G.H.: SY 24 \\
Mueller-Lisse T.G.: CC 1221 \\
Mueller-Mang C.: SS 204 \\
Müller-Schimpfle M.: RC 1902 \\
Munneke G.J.: SS 17016
\end{tabular}

\section{N}

Nadrljanski M.: SS 1302

Natale L.: SS 503

Nijenhuis R.: SS 609b

Nyhsen C.M.: SS 1801b

$\mathbf{0}$

Oleaga Zufiría L.: SS 1711

Ollivier L.: SS 902

Olsen Ø.E.: RC 1912

Omoumi P.: SS 610b

Ording-Müller L.-S.: SS 912

Oyen R.H.: SS 1307

$\overline{\text { P }}$

Padovani R.: SS 213

Padrón M.: RC 1410

Panicek D.M.: MC 428

Papadaki E.: SS 1811a

Papathanasiou M.A.: RC 1511

Paprottka P.M.: SS 109

Parkar A.P.: SS 1704

Patak M.A.: RC 1601b

Paulo G.: EM 5

Peebles C.: RC 803

Pekarovic D.: RC 1214

Pellegrin A.: SS 1815

Pellerin O : SS 215

Pereira P.L.: CC 1519

Petakovic A.: SS 914

Pickuth D.: $S Y 8$

Pierot L.: SS 509

Pinochet M.A.: EM 4

Piscaglia F: Joint Session of the ESR and EFSUMB

Plagou A.: SS 1310

Popovic P.: SS 201a

Prayer D.: $E^{3} 520 \mathrm{C}$

Prevoo W.: CC 1419

Prokop M.: RC 1513, SY 11

Pronk-Larive D.: RC 714 
Q

Quaia E.: SS 610a

$\mathbf{R}$

Raissaki M.: $R C 1512$

Rees M.R.: SS 603a

Regge D.: SS 905

Reiser M.F.: MC 728

Ribeiro R.: SS 114

Richenberg J.: SS 107

Ringertz H.: SS 1712

Rockall A.G.: SS 607

Roding T:: SS 114

Rollandi G.A.: RC 1201

Ros P.R.: SS 606

Ruiz Blanco C.: EM 5

Rummeny E.J.: SS 516

\section{S}

Sala E.: CC 1321

Sanz-Requena R.: SS 103

Saranovic D.Z.: SS 1701a

Sardanelli F.: MC 623

Schaefer-Prokop C.M.: SS 1704

Schönberg S.O.: SY 14

Schroth G.: SS 211

Schueller G.: SS 1317

Schuknecht B.F.: SS 1308

Schüller-Weidekamm C.: SS 110

Schulz-Wendtland R.: SS 102

Sconfienza L.M.: SS 210

Sekovski B.: SS 1709

Semmler W.: RC 1906

Shahabpour M.: SS 110

Sharp P.: EF 1

Sidhu P.S.: $S Y 9$

Sigal R.C.: SY 13

Simisker A.: SS 105

Sinitsyn V.E.: MC 422, SY 21

Skaane P.: SS 502

Sklair-Levy M.: SS 1802a

Smith A.: SY 17

Souquet J.: $S Y 2$

Speck O.: SS 113

Sperryn C.W.: EM 3

Steens S.: SS 208

Stenzel M.: SS 1812

Stern E.J.: SS 1304

Stiller W.: SS 1813

Sugimura H.: SS 1707

Šurlan Popovič K.: RC 1508

Sutton D.G.: RC 1413

Syrgiamiotis V.: SS 514

Szabó E.: SS 1802b

\begin{tabular}{l}
\hline \\
\hline Tacelli N.: SS 104 \\
Tack D.: SS 1301 \\
Tacke J.: SS 909 \\
Taibbi A.: SS 1301 \\
Tali E.T.: RC 1911 \\
Tardivon A.: MC 723, RC 1402 \\
Telesca M.: SS 102 \\
Thurnher M.M.: CC 1118 \\
Torresin A.: EF 2 \\
Trianni A.: RC 1113 \\
Tsapaki V.: RC 1513 \\
Tsetis D.K.: SS 1309 \\
Tyurin I.E.: SY 20 \\
Tzarouchi L.C.: SS 911 \\
\hline \\
\hline
\end{tabular}

Vahtramae P.: SS 1714

Valdés Solís P.: RC 417

Van Beers B.: SS 601

van der Putten W.J.M.: EF 1

van Persijn van Meerten E.L.: CC 719

Vandulek C.: SS 514

Vassileva J.N.: EF 2

Venstermans C.: RC 411

Vernooij M.: SS 1711

Vieira A.: SS 1710

Vilela P.: SS 911

Villeirs G.M.: RC 1207

Vivas I.: SS 1807

Vlahos J.: SS 904

Vliegenthart R.: RC 1603

Vock P.: ESR Radiation Protection Session

Vogl T.J.: SY 12

Votrubová J.: RC 1413

\begin{tabular}{ll}
\hline & W \\
\hline Weishaupt D.: SS 1701b & \\
Widelec J.: SS 608 & \\
Wildberger J.E.: SY 10 & \\
\hline & Y \\
\hline Yule A.: SS 214 &
\end{tabular}

Zamboni G.A.: SS 901

Zelenak K.: SS 909

Zimny A.: SS 1311

Zytoon A.A.: SS 202 